\title{
Article \\ Identifying Energy Extraction Optimisation Strategies of Actinobacillus succinogenes
}

\author{
Waldo Gideon Lexow, Sekgetho Charles Mokwatlo, Hendrik Gideon Brink (1) and Willie Nicol * \\ Department of Chemical Engineering, University of Pretoria, Lynnwood Road, Hatfield, Pretoria 0002, \\ South Africa; waldo.lexow@gmail.com (W.G.L.); mokwatlo.sekgetho@up.ac.za (S.C.M.); \\ deon.brink@up.ac.za (H.G.B.) \\ * Correspondence: willie.nicol@up.ac.za; Tel.: +27-420-3796
}

Citation: Lexow, W.G.; Mokwatlo, S.C.; Brink, H.G.; Nicol, W. Identifying Energy Extraction Optimisation Strategies of Actinobacillus succinogenes. Catalysts 2021, 11, 1016. https://doi.org/ $10.3390 /$ catal11081016

Academic Editors: Zheng Guo and Bekir Engin Eser

Received: 16 July 2021

Accepted: 20 August 2021

Published: 23 August 2021

Publisher's Note: MDPI stays neutral with regard to jurisdictional claims in published maps and institutional affiliations.

Copyright: (c) 2021 by the authors. Licensee MDPI, Basel, Switzerland. This article is an open access article distributed under the terms and conditions of the Creative Commons Attribution (CC BY) license (https:/ / creativecommons.org/licenses/by/ $4.0 /)$.

\begin{abstract}
A. succinogenes is well known for utilising various catabolic pathways. A multitude of batch fermentation studies confirm flux shifts in the catabolism as time proceeds. It has also been shown that continuous cultures exhibit flux variation as a function of dilution rate. This indicates a direct influence of the external environment on the proteome of the organism. In this work, ATP production efficiency was explored to evaluate the extent of bio-available energy on the production behaviour of $A$. succinogenes. It was found that the microbe successively utilised its most-to-least efficient energy extraction pathways, providing evidence of an energy optimisation survival strategy. Moreover, data from this study suggest a pyruvate overflow mechanism as a means to throttle acetic and formic acid production, indicating a scenario in which the external concentration of these acids play a role in the energy extraction capabilities of the organism. Data also indicates a fleeting regime where $A$. succinogenes utilises an oxidised environment to its advantage for ATP production. Here it is postulated that the energy gain and excretion cost of catabolites coupled to the changing environment is a likely mechanism responsible for the proteome alteration and its ensuing carbon flux variation. This offers valuable insights into the microbe's metabolic logic gates, providing a foundation to understand how to exploit the system.
\end{abstract}

Keywords: Actinobacillus succinogenes; fermentation; energy; succinic acid

\section{Introduction}

Homogenous compound production is regarded as the holy grail in chemical conversion. This principle is no different when it comes to the use of microbial cell catalysts and is a key hurdle to help facilitate the transition to a bio-based chemical production economy. As is often the case in microbial-catalysis, maximum theoretical yields are not attained due to mixed acid fermentative metabolic behaviours inherent in many strains of microbes. One such an example is that of the bovine rumen bacterium Actinobacillus succinogenes. The microbe is best known for its competitive production of succinic acid from a variety of substrates [1], tolerance of high substrate and acid titers [2], and is consequently considered a top contender for industrial application. Furthermore, A. succinogenes' fermentative production of succinic acid, via a reductive TCA pathway, necessitates the incorporation of a molar equivalent of carbon dioxide, incentivising the routes' economic and environmental value [3]. However, its reductive pathway also necessitates a net consumption of redox, which is supplied by the organism through the activation of complementary redox producing pathways. These pathways, in the case of $A$. succinogenes, lead to the co-production of acetic, formic, and pyruvic acid. These acids not only divert carbon away from succinic acid production but also augment downstream processing costs.

Succinic acid bears remarkable structural similarity to maleic anhydride, a commodity chemical with an industrial market valued at 2.77 billion USD for the year of 2018 [4], and has been flagged by the US Department of Energy (DOE) as one of 12 key bio-derivable compounds that would help facilitate the aforementioned shift $[5,6]$. Moreover, recently, 
biobased production studies of succinic acid have demonstrated high succinic acid titers of $>100 \mathrm{~g} / \mathrm{L}$ using various microorganisms [7,8], with the highest for A. succinogenes being $98.7 \mathrm{~g} / \mathrm{L}$ [9]. Thus, the process shows promise for industrial scale application. This, however, is only possible if its bio-based production becomes cost-competitive with that of the more traditional petrochemical production route. Aspects such as finding cheaper feedstock [10], and attaining high SA productivity in high cell density fermentation [11] have been considered in literature in an effort to make bio-based production cost competitive. Additionally, of equal value would be the circumventing of by-product production altogether.

Near homogenous production of succinic acid by A. succinogenes is theoretically possible when considering the redox producing oxidative pentose phosphate pathway (OPPP) [12]. By liberating half a molar equivalent of carbon dioxide, enough redox may by produced to satisfy homogenous succinate production at a theoretical maximum of 1.124 g.g $\mathrm{g}^{-1}$ dextrose. Although this combination of catabolic pathways is favourable from a succinic acid production perspective, virtually all the literature regarding wild type A. succinogenes fermentation reports the accumulative presence of formic and acetic acid. Moreover, an acid distribution profile shift—evident in batch data and its direct association with dextrose consumption rate found in continuous production data [13] - points to an adapting catabolic flux.

This research looked toward obtaining a better understanding of the reasoning behind the organism's fermentative behaviour as it relates to an energy-motivated incentive. To this end, the study investigated the transient metabolic fluxes of $A$. succinogenes under batch conditions utilizing two distinct media (Media A \& Media B) representing rich and minimal media, respectively. The transient metabolic states of the organism were determined by solving the metabolic flux model and, consequently, the ATP generation as it relates to the catabolite and biomass concentrations in the reaction volume. In this regard, its findings may be invaluable in improving fermenter design and operation for obtaining succinic acid yields closer to the theoretical maximum. Moreover, the development of comprehensive physiological theories provides insight to aid in genetic alteration strategies for the more effective construction of productive designer microbial catalysts.

\section{Results}

Batch fermentations were performed using two separate growth media-growth media $\mathrm{A}$ and $\mathrm{B}$ chosen as model media representing rich and minimal growth media, respectively. This was done to assess the effect of growth media on the energy incentivized fermentative behavior of the microorganism. For each growth medium two batch runs were performed for repeatability.

Samples analysed by HPLC were fitted with asymmetric (five parameter) or bi-logistic curves (depending on goodness of fit), with the bottom parameter constrained to zero. Curves fitted generally presented an $\mathrm{R}$ squared parameter of $>0.99$; however, a value $>0.95$ was deemed representative (Figure 1 and Table 1). Both media A and media B displayed similar behavioural trends amongst repeat batches and were considered to be demonstrative of the particular conditions exposed to them.

Both media tested (The choice and composition of media are discussed in detail in the Materials and Methods section.) repeatably displayed the initial phases of logistic growth followed by an extensive and erratic reduction in the measured biomass concentration. This phenomenon was correlated to the onset of flocculation-a brief period in which the measured concentration became less stable but was still increasing-and eventual biofilm deposition, resulting in a reduction of measured biomass. To circumvent this complication, the precipitated biofilm was resuspended and homogenised in the liquid broth post experimental run, after which measurements of DCW were made in triplicate to fix the final biomass concentration. To this single data point and high-resolution data of the initial stages of platonic growth was fitted a four-parameter logistic curve to gain an accurate estimate representation of the biomass accumulation. Clear repeatable logistic 
curves were obtained for both media A and B, though there was a time delay for media B. This time-delay shift, of approximately $4 \mathrm{~h}$, was most likely the cause of a lower initial starting concentration of viable cells in the inoculum.

Media A consistently displayed higher accumulation of succinic (Suc), acetic (Ace) and formic (For) acid compared to media B (Table 1). One key observed difference was found in the amount of measured pyruvic acid (Pyr). Media B resulted in the excretion of nearly twice the amount of pyruvic acid compared to media A. Furthermore, pyruvic acid was found to be the second most abundant acid present in media $B$ after succinic acid. Additionally, pyruvic acid exertion rates in media A were nonconforming between the two runs; the repeat run displayed less of a rapid accumulation profile of pyruvic acid. No measurable accumulation of ethanol, oxaloacetic, malic, fumaric, or lactic acid was evident. Run 1 of media A and run 2 of media B were selected to showcase further analysis because these were the only runs with accompanying oxidoreduction potential (ORP) data.

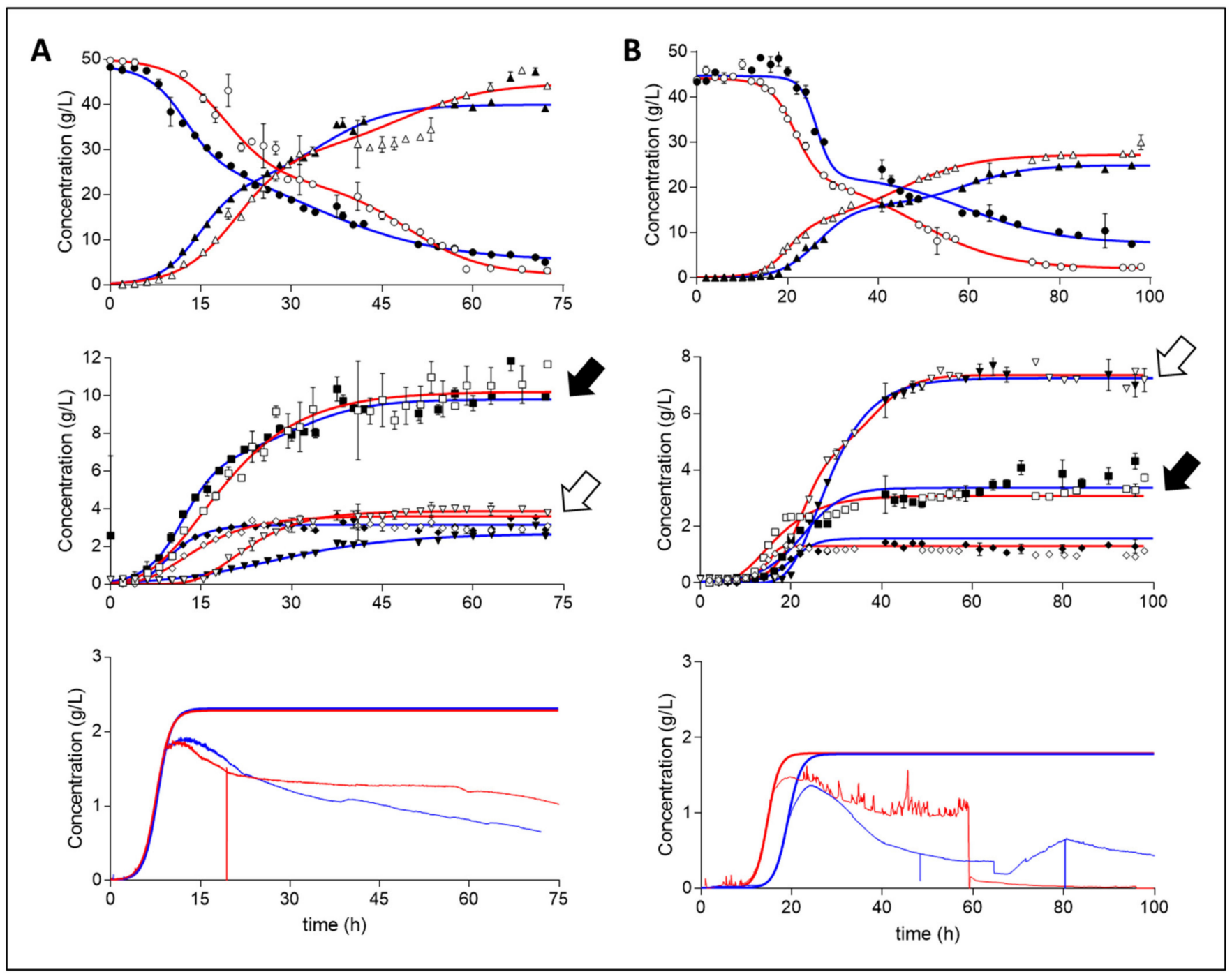

Figure 1. Repeat batch concentration profiles (g/L) of A. succinogenes on media (A) and media (B). Legend: dextrose (Dex), $\bigcirc$; succinic acid (Suc), $\triangle$; acetic acid (Ace), $\square$; formic acid (For), $\diamond$; pyruvic acid (Pyr), $\sigma$; repeats are indicated as filled shapes with blue fits. Biomass profiles (bottom) display online $\mathrm{OD}_{660}$ absorbance data and their logistic fits, with the top parameter fixed to a final DCW determined concentration. Black arrows (acetic acid) and white arrows (pyruvic acid) highlight the shift in their relative abundance between the two media. 
Table 1. The final titres $\left(\mathrm{g} \mathrm{L}^{-1}\right)$ and goodness of fit $\left(\mathrm{R}^{2}\right)$ of each respective fitted curve at fermentation termination.

\begin{tabular}{clllll}
\hline & & A-1 & A-2 & B-1 & B-2 \\
\hline \multirow{2}{*}{ Succinic acid } & $\mathrm{g} \mathrm{L}^{-1}$ & 44.62 & 39.89 & 27.20 & 24.87 \\
& $\mathrm{R}^{2}$ & 0.9978 & 0.9984 & 0.9987 & 0.9977 \\
Pyruvic acid & $\mathrm{g} \mathrm{L}^{-1}$ & 3.870 & 2.766 & 7.363 & 7.252 \\
& $\mathrm{R}^{2}$ & 0.9863 & 0.9886 & 0.9980 & 0.9958 \\
Acetic acid & $\mathrm{g} \mathrm{L}^{-1}$ & 10.22 & 9.802 & 3.081 & 3.369 \\
& $\mathrm{R}^{2}$ & 0.9645 & 0.9935 & 0.9737 & 0.9590 \\
Formic acid & $\mathrm{g} \mathrm{L}^{-1}$ & 3.604 & 3.145 & 1.312 & 1.577 \\
& $\mathrm{R}^{2}$ & 0.9937 & 0.9985 & 0.9933 & 0.9945 \\
Biomass & $\mathrm{g} \mathrm{L}^{-1}$ & 3.272 & 3.300 & 2.640 & 2.700 \\
& $\mathrm{R}^{2}$ & 0.9995 & 0.9976 & 0.9848 & 0.9888 \\
Dextrose & $\mathrm{g} \mathrm{L}^{-1}$ & 2.172 & 5.293 & 2.047 & 7.581 \\
& $\mathrm{R}^{2}$ & 0.9949 & 0.9991 & 0.9996 & 0.9866 \\
\hline
\end{tabular}

Redox balances, based on the metabolic pathway analysis of NADH consuming and producing metabolites, displayed a similar trend across both media compositions (Figure 2). Volumetric rate analysis revealed a scenario in which NADH was being produced at a faster rate than it was consumed during the initial stages of growth. This was followed by regime of near-to-complete NADH balance closure (uncertainty due to an accumulative error of variance in metabolite fits), and then by a phase in which more NADH was being consumed than could be accounted for alone by the NADH producing metabolites measured. Initial stages of higher NADH production rates coincided with a drop in the media oxidoreduction potential. In contrast, the final stages of the fermentation displayed an over-consumption of NADH (based on measured metabolites), providing a clear indication of an unmeasured $\mathrm{NADH}$ producing metabolite. Here, it is thought that the oxidative pentose phosphate pathway (OPPP) is the likely culprit because the pathway results in no additional catabolites other than $\mathrm{CO}_{2}$ (Bradfield and Nicol, 2016). Because the production of succinic acid entails the incorporation of $\mathrm{CO}_{2}$, the reactor was sparged with $\mathrm{CO}_{2}$ to saturate the environment. Due to this, gas analysis could not be performed to verify OPPP activity. However, because no additional catabolites were measured in the liquid phase, the OPPP seems the likely explanation. This suggests a state of homosuccinic acid production toward the end of batch fermentation.

Mass balances were performed to establish if mass closure could provide supporting evidence toward OPPP activity by assuming that the unmeasured redox production came from the OPPP. Media B exhibited a near-to-complete carbon closure (Uncertainty due to an accumulative error of variance in metabolite fits), whereas media A presented more $\mathrm{cmol}$ metabolites produced than cmol dextrose consumed. This deviation, however, is small enough for the carbon gain to be explained by the additional source of carbohydrate and amino acid compounds present in the $10 \mathrm{~g} \mathrm{~L}^{-1}$ corn steep liquor (CSL) and $6 \mathrm{~g} \mathrm{~L}^{-1}$ yeast extract (YE) in addition to the dextrose in media A. Although mass balance closure could not be demonstrated in media $\mathrm{A}$, the closure in media $\mathrm{B}$ suggests that the redox imbalance observed toward the end of fermentation is most likely the cause of OPPP activity. This is because, if an unmeasured net NADH-producing catabolite has been the complement to the redox imbalance observed, the mass balance would have predicted less dextrose (Dex) consumed than was measured. 
A1

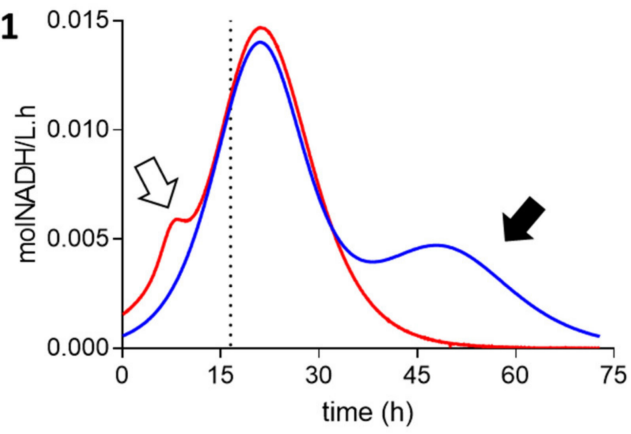

A2

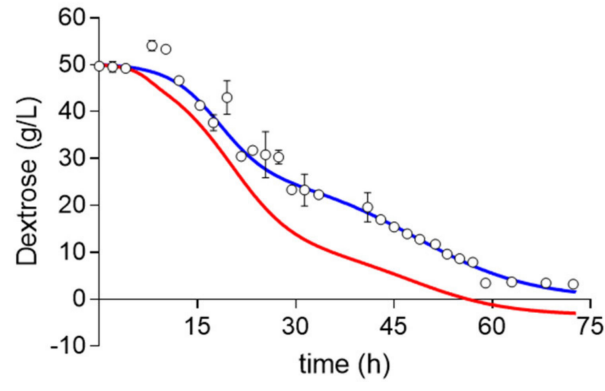

B1

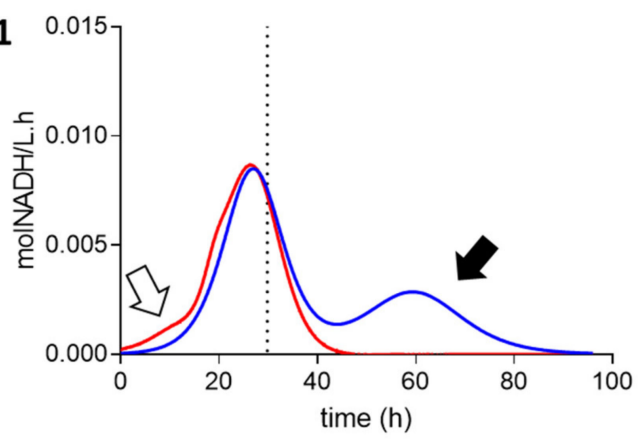

B2

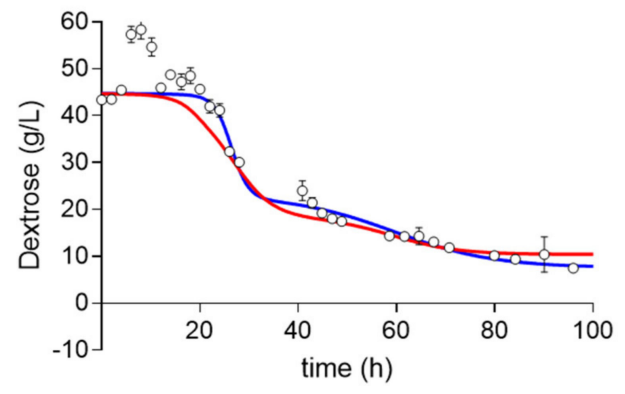

Figure 2. Fermentation redox and mass balances of Media A and Media B. Redox balances (A1,B1): Volumetric NADH consumption rate (blue) and production rate (red) as calculated from the measured catabolites (Suc, Pyr, Ace, For). White arrows highlight net NADH production with no measured consuming counterpart, whilst black arrows highlight a net measured redox production with no measured redox producing counterpart. Dotted line signifies the termination of growth. Mass balances (A2,B2): Measured dextrose concentration (circles) and their fits (blue) are compared to the predicted glucose concentration (red) as determines from the measured metabolite concentrations. A near to accurate closure is obtained in media B whilst media A continuously displays lower dextrose consumption than is predicted, attributed to the additional carbohydrate content of CSL and YE.

\section{Discussion}

Batch fermentation on Actinobacillus succinogenes typically yields a shifting product distribution profile as fermentation progresses. Furthermore, it has previously been reported by this research group that the product distribution profiles changed with increasing dextrose consumption when measured in a continuous bioreactor [13]. Here it was reported that concentration of Suc increased with an increasing dextrose consumption, whereas the concentration of acetic and formic acid both increased initially and then subsequently flattened out or steadily decreased to zero, respectively. For this to occur, changes in metabolic strategies take place in which one enzymatic route is newly favoured over another in order to achieve a state of homeostasis. One consideration is that these changes are the result of an energy optimisation mechanism exploited by the organism to thrive in an increasingly harsh environment. To explore this idea, a comprehensive grasp of energy production efficiency is warranted.

\subsection{Theoretical Analysis of Energy Production Strategies}

The production of succinic acid in wild-type A. succinogenes occurs via a reductive branch of the TCA cycle (Figure 3). This is due to the organism lacking both a complete TCA cycle and a glyoxylate shunt that would endow the organism with the ability to produce it oxidatively [14]. By producing succinic acid in such a way A. succinogenes requires a molar equivalent of reduction power (Equation (1)).

$$
1 / 2 \mathrm{Dex}+\mathrm{CO}_{2}+\mathrm{NADH} \rightarrow \mathrm{Suc}(+5 / 3 \mathrm{ATP})
$$




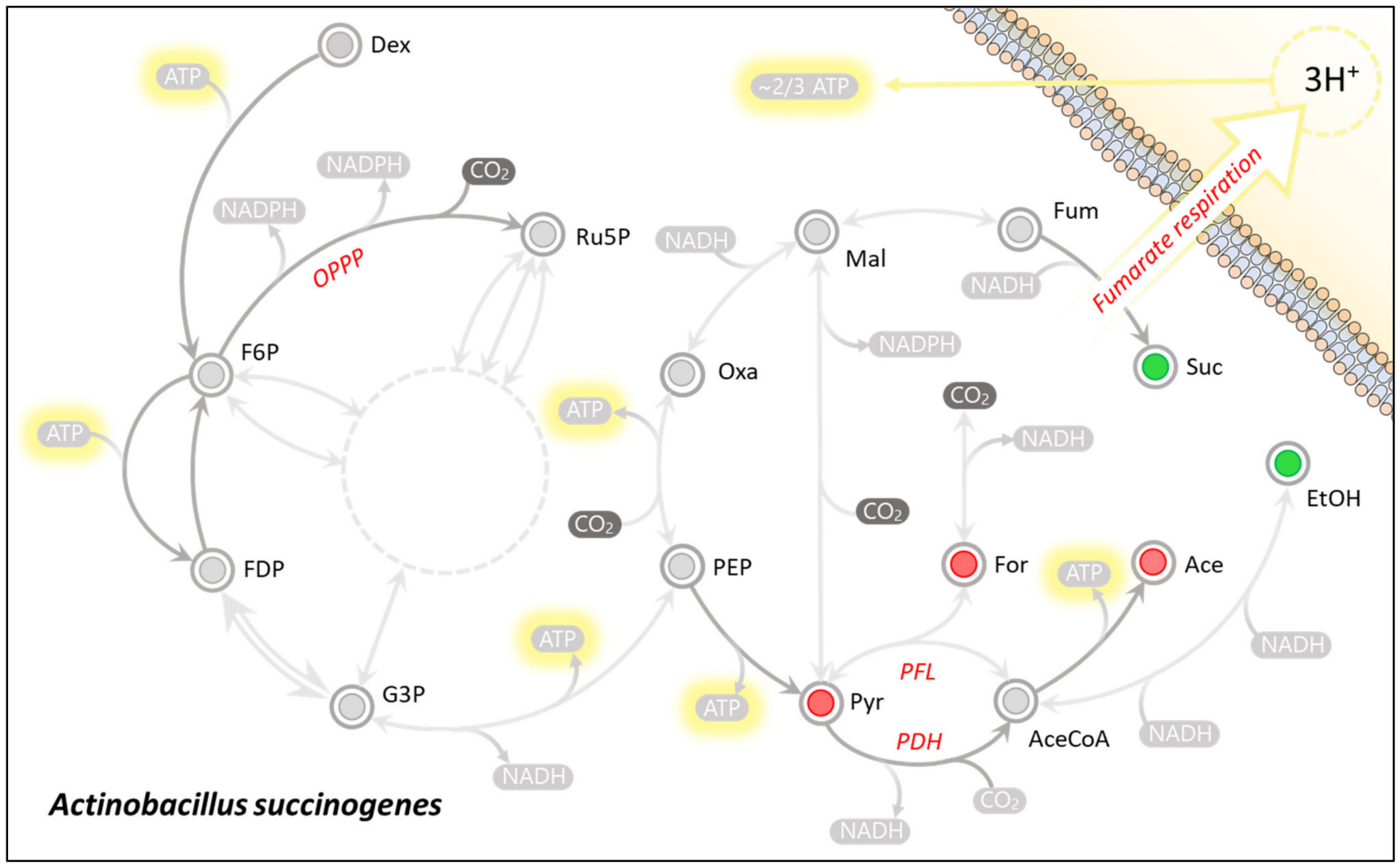

Figure 3. Main nodes of Actinobacillus succinogenes central carbon metabolism. Unidirectional arrows (dark) indicate proposed unidirectional fluxes [3], where all other fluxes are considered to be reversible. The dotted circle represents the pentose phosphate pathway overall reaction of the transketolase carbon exchange mechanism simplified to $3 \mathrm{Ru} 5 \mathrm{P} \rightleftharpoons 2 \mathrm{~F} 6 \mathrm{P}+$ G3P. The Red nodes (incl. For, Ace, and Pyr) highlight metabolites which are net NADH producing metabolites, whereas green nodes (inc. Suc and $\mathrm{EtOH}$ ) highlight net NADH consuming metabolites.

In order to neutralise the redox imbalance caused by the succinic acid production, the redox deficit needs be sourced from a complementary redox producing pathway. The organism is known to accomplish this by diverting carbon flux from the pyruvate node toward the oxidative production of acetic acid (Figure 3). In this case, pyruvate may either be catabolised by Pyruvate-Formate-Lyase (PFL) or Pyruvate-Dehydrogenase (PDH) [11]. This results in the respective liberation of either formic acid or $\mathrm{CO}_{2}$ in addition to acetylCoA, which is subsequently converted to acetic acid (Equations (2) and (3)).

$$
\begin{gathered}
\text { PFL: } 1 / 2 \text { Dex } \rightarrow \text { Ace }+ \text { For }+ \text { NADH (+2ATP) } \\
\text { PDH: } 1 / 2 \text { Dex } \rightarrow \text { Ace }+\mathrm{CO}_{2}+2 \mathrm{NADH}(+2 \mathrm{ATP})
\end{gathered}
$$

Though both pathways result in similar ATP yields, the PDH pathway produces twice the amount of NADH compared to PFL. Therefore, to compare energy production strategies between the two, it is beneficial to consider energy production as part of a redox neutral route, normalised to a common base. With succinic acid production as the redox consuming branch and PFL as the redox producing branch, and assuming the conversion of fumaric acid to succinic acid results in a static $2 / 3$ mol ATP gain, this energy production redox neutral pathway produces $11 / 3 \mathrm{~mol}$ ATP for every $6 \mathrm{cmol}$ dextrose consumed (0.611 mol ATP/cmol Dex) (Figure 4). Similarly, with PDH as the redox producing branch, an amount of $16 / 3 \mathrm{~mol}$ ATP is obtained for every $9 \mathrm{cmol}$ dextrose $(0.593 \mathrm{molATP} / \mathrm{cmolDex})$ (Figure 4). It, therefore, becomes apparent from these calculations that in terms of ATP yield per substrate consumed, the PDH pathway liberates slightly less energy compared to 
PFL ( 97\%). Formate-Dehydrogenase (FDH) activity was also found to be present in cell extracts [14] (Equation (4)).

$$
\text { For } \rightarrow \mathrm{CO}_{2}+\mathrm{NADH}
$$

Its activity coupled to the PFL route results in an identical energy liberation efficiency to that of the PDH route ( $0.593 \mathrm{~mol}$ ATP/ $\mathrm{cmol}$ Dex). Besides acetic acid and formic acid production, A. succinogenes has also been reported to secrete pyruvate (Equation (5)).

$$
1 / 2 \mathrm{Dex} \rightarrow \mathrm{Pyr}+\mathrm{NADH}(+\mathrm{ATP})
$$

Substituting pyruvate as the redox producing compliment an amount of $8 / 3 \mathrm{~mol}$ ATP is obtained per $6 \mathrm{cmol}$ dextrose $(0.444 \mathrm{~mol} \mathrm{ATP} / \mathrm{cmol}$ Dex) (Figure 4$)$. This approximates to $73 \%$ of energy that could have been captured if the PFL route was followed through. Furthermore, a case has also been made for the Oxidative Pentose Phosphate Pathway (OPPP) as a redox producing complement (Equation (6)).

$$
1 / 6 \text { Dex } \rightarrow \mathrm{CO}_{2}+2 \mathrm{NADPH}(-1 / 6 \text { ATP })
$$

Here too the energy gained per consumed substrate is significantly lower when compared to either of the acetic acid production strategies, yielding only 19/6 mol ATP gained per $7 \mathrm{cmol}$ dextrose consumed ( $0.452 \mathrm{~mol} \mathrm{ATP} / \mathrm{cmol}$ Dex) (Figure 4$)$, roughly $74 \%$ of that produced by PFL as the redox producing complement. Interestingly, when supplied with a theoretical free external source of redox [15], homosuccinic acid production will occur at $0.556 \mathrm{~mol}$ ATP $/ \mathrm{cmol}$ Dex, which still is less energy that would have been gained if acetic acid production was in effect.

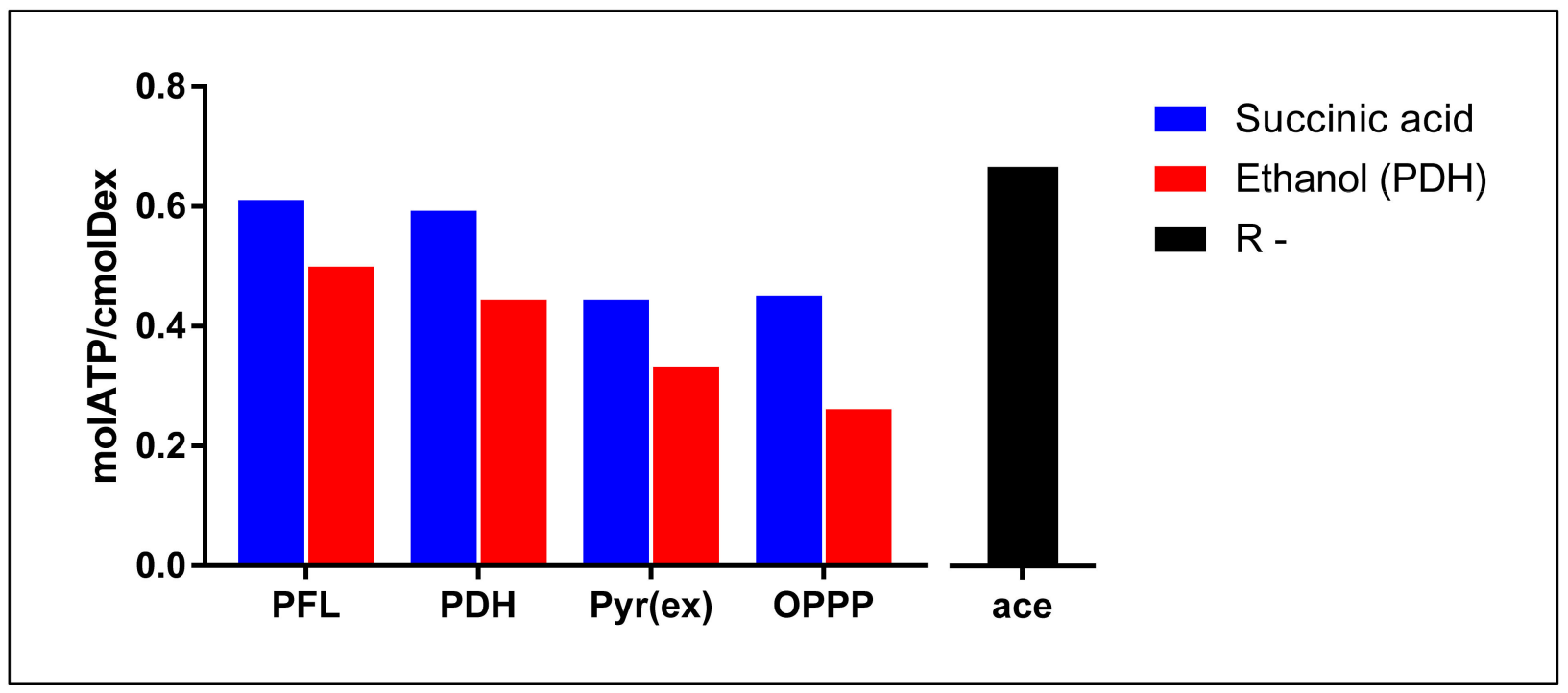

Figure 4. Visual comparison of static energy extraction efficiencies of PFL, PDH, Pyruvate excretion, and OPPP when coupled to succinic acid as redox consuming counterpart (blue) or ethanol as redox consuming counterpart (red). R-: energy extraction efficiency when reduction power is lost to the environment, producing an acetic acid (black). These numbers result from calculations based on metabolic pathways and not from experimental measured data using enzyme essays.

\subsection{On Ethanol Production}

Besides succinic acid, A. succinogenes also maintains the ability to produce ethanol $(\mathrm{EtOH})$ as a redox consuming compliment when produced with the aid of the PFL route (Equation (7)).

$$
\text { 1/2Dex }+\mathrm{NADH} \rightarrow \mathrm{EtOH}+\text { For }(+\mathrm{ATP})
$$

A quick glance at energy limitations highlights why succinic acid is favoured above ethanol production. When combined with the PFL redox producing route (Equation (2)) 
an amount of $0.5 \mathrm{~mol}$ ATP/cmolDex is extracted (Figure 4 ). This covers only $82 \%$ of the energy gained when succinate production is the redox consuming branch. Likewise, the $\mathrm{PDH}$, Pyruvate excretion, and OPPP redox producing routes, when coupled with ethanol production via the PFL route, results in 75\% (0.444 mol ATP/cmol Dex), 75\% (0.333 mol ATP/cmol Dex), and 58\% (0.262 mol ATP/cmol Dex) of the energy that would have been gained if succinic acid had been the redox consuming compliment, respectively (Figure 4). Ethanol may also be produced as a redox neutral product when produced via the PDH route (Equation (8)).

$$
1 / 2 \mathrm{Dex} \rightarrow \mathrm{EtOH}+\mathrm{CO}_{2}(+\mathrm{ATP})
$$

This method too results in low ATP production efficiency at $0.333 \mathrm{~mol} \mathrm{ATP} / \mathrm{cmol}$ Dex. These efficiencies emphasize why ethanol production is low or lacking in many fermentation studies regarding $A$. succinogenes because, at its best, it only supplies $82 \%$ of the energy that succinic acid would supply. It would seem the organism primarily retains the ability to produce EtOH as a failsafe to an obstruction of succinic acid production, such as when $\mathrm{CO}_{2}$ mass transfer is insufficient, in order to rid itself of toxic redox build-up [16]. Furthermore, it may serve to support succinic acid production by recycling $\mathrm{CO}_{2}$ generated by ethanol production in order to obtain better ATP yields.

\subsection{Energy Extraction Pathway Relay}

Although this simple analysis provides support as to why A. succinogenes fermentation starts off with a near stellar 1:1:1 ratio production of succinic, acetic and formic acid-from an energy efficiency perspective-it does not offer clarity on why a metabolic shift occurs. To expand on this idea, the high-resolution batch fermentation profiles were generated to elucidate if a consistent chronology could be found (Figure 1).

Once redox and mass balances were confirmed, individual redox neutral energy pathway activities were established by proportioning molar volumetric rates to the abovementioned combinatory pathways (Figure 5). By partitioning the acid production rates in their respective redox neutral pathways, a clear pattern, irrespective of media composition, is apparent. From the data, it is evident that, except for pyruvate excretion, $A$. succinogenes successively cycles through its most (ORP: $0.667 \mathrm{~mol} \mathrm{ATP/cmol} \mathrm{Dex)} \mathrm{to} \mathrm{its} \mathrm{least} \mathrm{(OPPP:}$ $0.452 \mathrm{~mol}$ ATP/cmol Dex) efficient energy extraction route.

Initially, when regarding succinic acid as the sole NADH sink, the PFL route is actively producing a 1:1:1 ratio of succinic, acetic, and formic acid. This is followed by a shift toward a regime in which formic acid production is omitted, resulting in a 2:1 production ratio of succinic to acetic acid. From this data, it is unclear whether PFL activity is gradually replaced with that of $\mathrm{PDH}$ activity, or if FDH activity is steadily upregulated, or a combination of the two mechanism, all of which would provide the same response. For the purpose of this discussion, however, it will be referred to as $\mathrm{PDH}$ activity. The peak in PDH activity is correlated with a proximate peak in pyruvic acid excretion activity. Considering the huge difference in energy production efficiencies between PDH and pyruvic acid excretion pathways, an argument can be made for pyruvic acid production primarily operating as an overflow mechanism to divert carbon away from acetic acid production. This argument is supported by the finding that no measurable trace of oxaloacetic acid was present, the production pathway of oxaloacetic acid would result in an equivalent energy efficiency to that of pyruvic acid production at $0.444 \mathrm{~mol}$ ATP/cmol Dex. The co-activity of PDH and pyruvic acid excretion is followed by the shift toward complete OPPP activity, a homo-succinic acid production pathway. Of note is that $A$. succinogenes did not utilise energy production strategies that would result in lower energy production efficiencies than the OPPP could provide, such as the production of the redox neutral products ethanol, malic, fumaric or lactic acid, all of which result in an efficiency of $0.333 \mathrm{~mol}$ ATP/cmol Dex. This, however, is beneficial to the cause of succinic acid production because no carbon is lost to these additional catabolites. Furthermore, prior to the onset of succinic acid production, acetic acid production was already in effect. This denotes a regime in which succinate does not act as the redox consuming counterpart. 
As a reduction in the overall media ORP was observed, it is thought that the reduction potential is lost to, or used to condition, the aqueous environment in addition to the redox required by biomass formation [14,17] (Equation (9)).

$$
\mathrm{nCH}_{2} \mathrm{O}+\mathrm{mNH} 3+\omega \mathrm{NADPH}+\gamma \mathrm{ATP} \rightarrow \mathrm{X}+\alpha \mathrm{CO}_{2}+\beta \mathrm{NADH} ; \omega>\beta
$$

From an energy perspective, it conforms to the pattern of utilising more efficient routes first, as the uncoupled production of acetic acid results in an efficiency of $0.667 \mathrm{~mol}$ ATP / cmol Dex which exceeds the efficiency of PFL (Figure 4). Reduction of the media ORP was only observed to take place until the media reached and ORP of $-370 \mathrm{mV}$, similar to the findings of Li et al. [18]. This indicates a ceiling that prevents A. succinogenes from continuously utilising the media as a NADH sink to favour pure acetic acid production. This effect is exemplified in Li et al. [18] where the ORP was controlled at $-100 \mathrm{mV}$ bringing about a drastic shift in the acetic to succinic acid ratio. Because the aqueous environment continuously provided a way to utilise the media as a redox consumer, the acetic acid concentration accumulated as usual whilst succinic acid production was severely suppressed. Conversely, when conditions where controlled at $-350 \mathrm{mV}$, the highest succinic to acetic acid ratio was obtained as the media was less able to perform as a redox consuming counterpart to acetic acid production. Moreover, as indicated in (Figure 5), the termination of the media-assisted energy production pathway is closely associated with the onset of flocculation in both media compositions as well as an increase in the overall cell-based dextrose consumption rates, followed by a decrease once growth terminates. Flocculation occurs as the initial act in the biofilm formation phase; as such, biofilm was observed on the reactor walls soon after. The perturbation of the dextrose consumption rate witnessed is a conservative estimate as it is based on the fitted biomass curve; an even greater shift would be apparent should the measured data have been used to calculate cmolX $\mathrm{C}^{-1}$ based rates. This phenomenon is likely explained by an increase in the energy demand for biofilm formation.

Although it is evident from this analysis that A. succinogenes is continually diminishing its energy extraction efficiency, it should be pointed out that the process results in lower production ratios, and eventually the complete production cessation, of first formic and then acetic acid. This may imply a mechanism in which the external concentration of these acids provides enough of a stress response to induce the shutdown of their catabolic production by A. succinogenes. Such a mechanism becomes plausible when considering the fact that all produced catabolites must consume a fraction of the energy generated by their production in order for the cell to rid itself of their cytotoxic accumulation. The bi-lipid membrane remains a selective barrier preventing the transfusion of charged molecules, such as dissociated organic acids, a state in which all organic acids produced by $A$. succinogenes are in at a cytosolic $\mathrm{pH}$ of $>7.0$. Due to limited passive diffusion the cell may implement to rid itself of the cytotoxic build-up of these catabolites, an energy associated transport mechanism would be required. Furthermore, due to high permeability coefficients [19], it is plausible that formic and acetic acid may easily penetrate the bi-lipid membrane by means of passive back diffusion once sufficient extracellular concentrations are reached. Theoretically, this would result in an increasing ATP cost to excrete these catabolites vs energy gained from their production to maintain homeostasis, and subsequently generating the need to shut down their production. 


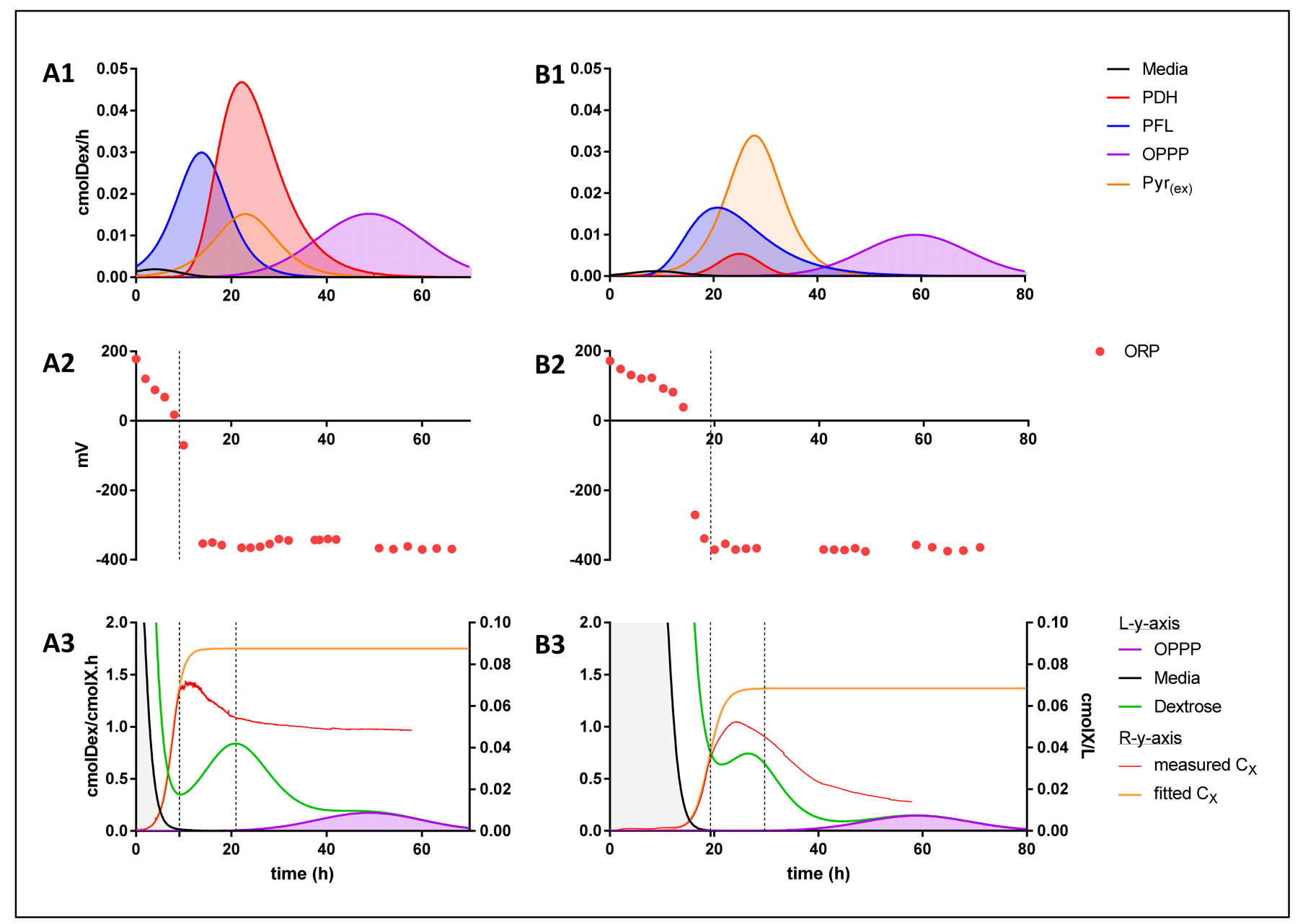

Figure 5. (A1,B1) Volumetric dextrose consumption rate as it relates to the specific energy production pathways utilised, both media display preference to pathways with higher energy extraction efficiencies, sequentially substituting them for pathways with lower energy extraction efficiencies as fermentation progresses. (A2,B2) Measured ORP data. (A3,B3) [left y-axis]) Depiction of cell-based dextrose consumption rates (green), and cell-based dextrose consumption rates as it relates to the media mediated (ORP) energy extraction pathway (grey) and OPPP energy extraction pathway (purple). [right $\mathrm{y}$-axis]) Measured (red) and fitted (orange) biomass concentration curves; A correlation coupling the onset of flocculation to termination of the ORP energy extraction pathway is obtained.

\section{Materials and Methods}

\subsection{Culture}

All chemicals were of reagent grade and purchased from Sigma-Aldrich (St. Louis, MO, USA) except for dextrose monohydrate, which was purchased form Bragan Chemicals (Johannesburg, South Africa), and $\mathrm{CO}_{2}(\mathrm{~g})$, obtained from Afrox (Johannesburg, South Africa). Actinobacillus succinogenes 130Z (DSMZ 22257; ATCC 55618), procured from the German Collection of Microorganisms and Cell Culture (DSMZ), was propagated in $100 \mathrm{~mL}$ Tryptic Soy Broth (TSB) at $37^{\circ} \mathrm{C}$ to an $\mathrm{OD}_{660}$ of 1.0 , at which time the culture was cryogenically stored $\left(-40^{\circ} \mathrm{C}\right)$ in $2 \mathrm{~mL}$ cryotubes, by suspending $0.5 \mathrm{~mL}$ of culture broth in $1 \mathrm{~mL}$ of glycerol. This was done to ensure genetic stability throughout experimentation. Prior to experimental start-up, cells were revived from cryogenics by allowing the stock to thaw to ambient temperature and subsequently inoculating it aseptically into $25 \mathrm{~mL}$ screwcap vials containing $20 \mathrm{~mL}$ TSB pre-heated to $37^{\circ} \mathrm{C}$. These starter cultures were then incubated for $16 \mathrm{~h}$ at $37^{\circ} \mathrm{C}$ and $250 \mathrm{rpm}$ after which $10 \mathrm{~mL}$ of the volume was transferred to the bio-reactor via a hypodermic needle. To assess reproducibility, individual experiments were successively performed in duplicate. 


\subsection{Growth Medium}

Two separate media were used in this study - growth media A and B-chosen as model media representing rich and minimal growth media, respectively. Growth media A consisted of (in $\mathrm{g} \mathrm{L}^{-1}$ final concentration) 50.0 dextrose, 10.0 clarified corn steep liquor (CSL), 6.0 yeast extract (YE), $0.2 \mathrm{MgCl}_{2} \cdot 6 \mathrm{H}_{2} \mathrm{O}, 0.2 \mathrm{CaCl}_{2} \cdot 2 \mathrm{H}_{2} \mathrm{O}, 1.0 \mathrm{NaCl}, 3.0 \mathrm{~K}_{2} \mathrm{HPO}_{4}$, $1.6 \mathrm{NaH}_{2} \mathrm{PO}_{4}, 0.31 \mathrm{Na}_{2} \mathrm{HPO}_{4}$. Growth media B consisted of (in $\mathrm{g} \mathrm{L}^{-1}$ final concentration) 50.0 dextrose, 1.7 yeast nitrogen base without amino acids, $0.3 \mathrm{YE}, 3.0 \mathrm{gL}^{-1}$ tryptic soy broth, $3.0 \mathrm{~K}_{2} \mathrm{HPO}_{4}, 1.6 \mathrm{NaH}_{2} \mathrm{PO}_{4}, 0.31 \mathrm{Na}_{2} \mathrm{HPO}_{4}, 1.0 \mathrm{NaCl}, 2.0 \mathrm{NH}_{4} \mathrm{Cl}, 10 \mathrm{mg} \mathrm{L}^{-1}$ biotin, $25 \mathrm{mg} \mathrm{L}^{-1}$ nicotinamide, $150 \mathrm{mg} \mathrm{L}^{-1}$ glutamic acid, $80 \mathrm{mg} \mathrm{L}^{-1}$ methionine, $80 \mathrm{mg} \mathrm{L}^{-1}$ cysteine (based on: McKinlay et al. [20]). Clarified corn steep liquor was prepared by heating a $200 \mathrm{~g} \mathrm{~L}^{-1}$ solution to $105{ }^{\circ} \mathrm{C}$ for $15 \mathrm{~min}$ and then decanting the supernatant once all solids have precipitated after cooling. Dextrose and phosphates were autoclaved separately from other media components $\left(121^{\circ} \mathrm{C}\right.$ for $\left.60 \mathrm{~min}\right)$, combining the mixtures aseptically once they had cooled down to ambient temperature. Components that could not be subjected to the extreme temperatures of the autoclaving procedure were added to the autoclaved mixture by means of filtration using a $0.2 \mu \mathrm{m}$ liquid filter (Midisart 2000, Sartorius, Göttingen, Germany).

\subsection{Fermenter}

The fermenter of choice was comprised of a $2.0 \mathrm{~L}$ reservoir jacketed-reactor vessel (1.5 L working volume), to which was coupled high flow liquid and gas peristaltic external recycle lines, respectively (Figure 6).

Both recycle lines were made of inert silicon tubing and served to agitate the liquid bulk to a homogenous composition. Furthermore, the liquid recycle line acted as a port for (i) an in-line aluminum $\mathrm{pH} /$ temperature probe station housing a Tophit CPS417D glass probe (Endress + Hauser, Gerlingen, Germany), (ii) a $10 \mathrm{M} \mathrm{NaOH}$ dosing inlet, (iii) an aseptic $\mathrm{CO}_{2}$ gas inlet, (iv) a sample outlet, and (v) a low liquid flow recycled bleedstream incorporated with an in-line spectrophotometric flow cell. Excessive foaming was controlled by dripping a 10\% (v/v) Antifoam SE-15 solution onto the liquid surface when needed. In addition, the vessel contained a septum sealed inoculation port, a $0.2 \mu \mathrm{m}$ PTFE filter fitted gas outlet port (Midisart 2000, Sartorius, Göttingen, Germany) to maintain atmospheric pressure, and a media inlet port which was used to fill the vessel prior to inoculation. Gas flow was controlled with a Brooks Mass Flow Controller (Hatfield, PA, USA), and $\mathrm{CO}_{2}$ was fed at $30 \%$ vvm to saturate the media and displace all dissolved $\mathrm{O}_{2}$. Heated water was continuously circulated to the heating jacket from a separate $5.0 \mathrm{~L}$ stirred vessel (4.0 L working volume) which was in direct contact with a PID-controlled heating plate. Fermentation was controlled at $37^{\circ} \mathrm{C}( \pm 0.6)$ and a pH of $6.8( \pm 0.05)$ by means of a Liquiline CM442 (Endress + Hauser, Gerlingen, Germany), coupled to a custom developed LabVIEW 2017 program (National Instruments), which toggled the output of the heating plate and peristaltic $\mathrm{NaOH}$ dosing pump on/off by means of a relay. Prior to start up the entire system (excluding the $\mathrm{NaOH}$ ) was autoclaved at $121^{\circ} \mathrm{C}$ for $60 \mathrm{~min}$, after which the reactor and sterilised feed were aseptically coupled by means of a custom-made stainless-steel $\mathrm{U}$ connector which was then sterilised by immersion in an oil bath $\left(140^{\circ} \mathrm{C}\right.$ for $30 \mathrm{~min}$ ). Media was then transferred to the reactor and allowed to stabilise at the desired $\mathrm{pH}$ and temperature before inoculation. 


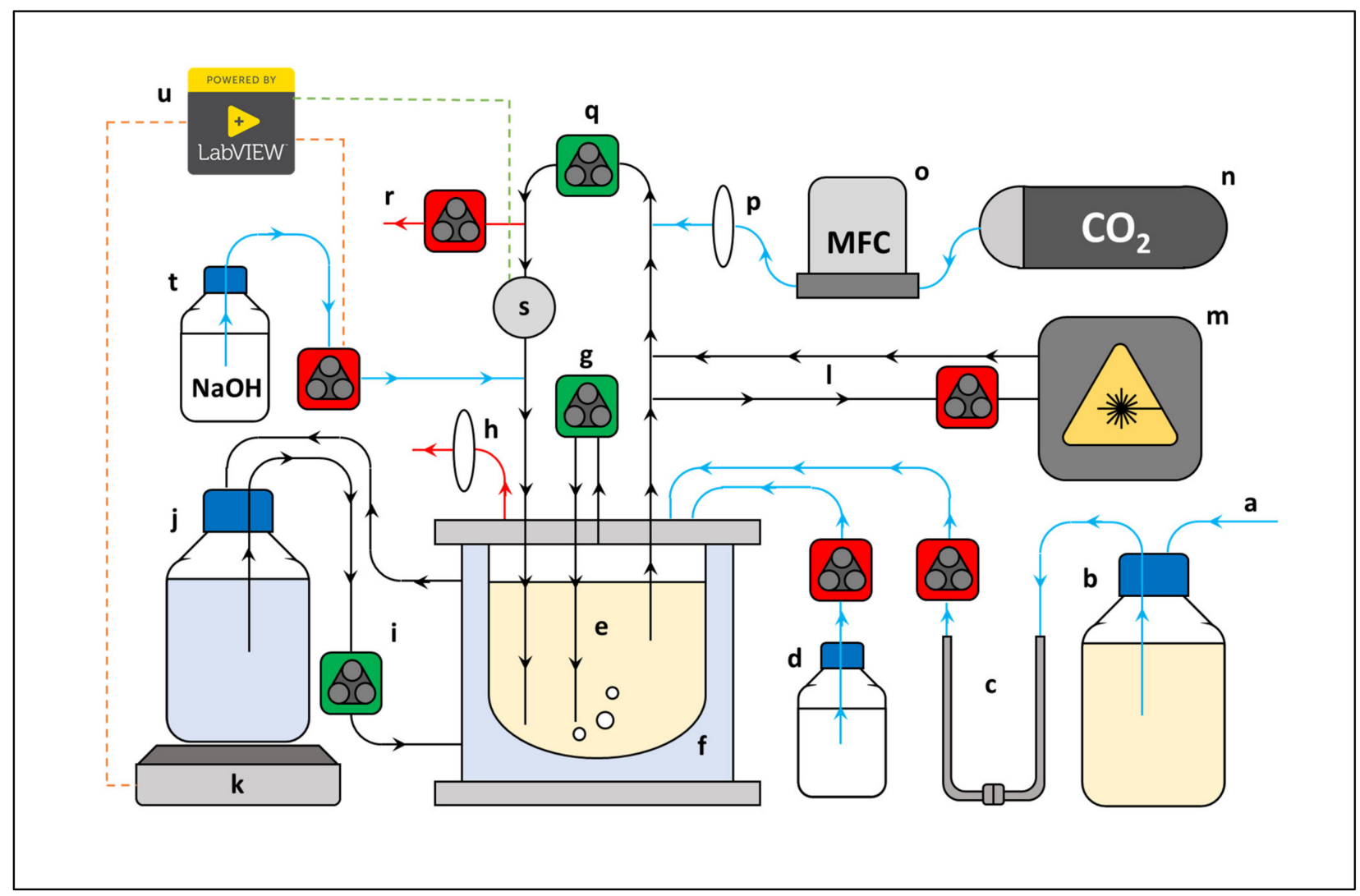

Figure 6. Simplified scheme of reactor setup. (a) multiple sterile inlet lines, (b) media reservoir, (c) detachable U-connection (sterilised in oil bath), (d) antifoam reservoir, (e) reactor vessel, (f) water jacket, (g) gas recycle line, (h) gas outlet, (i) heat transfer recycle line, $(\mathbf{j})$ heated water reservoir, $(\mathbf{k})$ hotplate, $(\mathbf{l})$ spectrophotometer recycle line, $(\mathbf{m})$ flow cell spectrophotometer, (n) $\mathrm{CO}_{2}$ gas tank, (o) mass flow controller, (p) air filter, (q) liquid recycle line, (r) sample line, (s) temperature/pH probe, (t) $\mathrm{NaOH}$ reservoir, (u) LabVIEW ${ }^{\mathrm{TM}}$ operated temperature/pH controller coupled to input (s), green stippled line) to subsequently regulate the outputs (t,k, red stippled lines). Pumps: high velocity (green), low velocity (red). Recycle lines (black), inlet lines (blue), outlet lines (red).

\subsection{Analytical Methods}

Over the experimental timespan $(70-100 \mathrm{~h}), 30 \times 2 \mathrm{~mL}$ samples were collected, including one at start-up $\left(\mathrm{t}_{0}\right)$, and placed on ice to halt metabolic activity. Samples were then filtered to remove cellular biomass after which their composition was determined by High Performance Liquid Chromatography (HPLC) Analysis, using an Agilent 1260 Infinity HPLC system (Agilent Technologies, Santa Clara, CA, USA) equipped with a $300 \times 7.8 \mathrm{~mm}$ Aminex HPX-87H ion-exchange column (Bio-Rad Laboratories, Hercules, CA, USA) and a Refractive Index detector. The mobile phase used for organic acid analysis consisted of $0.3 \mathrm{~mL} \mathrm{~L}^{-1} \mathrm{H}_{2} \mathrm{SO}_{4}$, and dextrose analysis was performed with a $1.5 \mathrm{~mL} \mathrm{~L}^{-1} \mathrm{H}_{2} \mathrm{SO}_{4}$ mobile phase. In either case, the flowrate was $0.6 \mathrm{~mL} \mathrm{~min}^{-1}$ with a column temperature of $60^{\circ} \mathrm{C}$. The samples were analysed in triplicate for each mobile phase condition and screened for the abundance of phosphates, dextrose, ethanol, glycerol, citric-, oxaloacetic-, malic-, fumaric-, succinic-, pyruvic-, acetic-, and formic acid. Moreover, lactic acid concentration was monitored, and its accumulation was used as a benchmark for infection, in which case the fermentation was immediately terminated. The phosphates peak was used as an internal standard because its profile remains constant throughout fermentation, so all other measured metabolites concentrations were adjusted accordingly to factor out the effect of dilution errors resulting from $\mathrm{NaOH}$ dosing and antifoam addition. Suspended Dry Cell Weight (DCW) accumulation was measured online using an Agilent Cary 60 UV-Vis Spectrophotometer (Agilent Technologies, USA) equipped with a Quartz Micro Flow Cell (10 mm pathlength; $70 \mu \mathrm{L}$ internal volume) connected in-line to the secondary 
liquid recycle line of the fermenter. High resolution growth profiles were obtained by collecting a one-second average measured at $\mathrm{OD}_{660}$ every $4.8 \mathrm{~s}$. Absorbance values were then converted to concentrations based on a function fitted to a standard curve determined for the DCW of A. succinogenes. Samples used for setting up the DCW-OD 660 correlation were centrifuged at $12,100 \times g$ for $90 \mathrm{~s}$, after which the cell pellets were washed twice with PBS solution, suspending and re-centrifuging the cellular biomass, and then dried for $48 \mathrm{~h}$ at $85^{\circ} \mathrm{C}$.

\subsection{Data Analysis}

Curve fitting, manipulation, and analysis of the data was done with the aid of GraphPad Prism 7.00 software (GraphPad Software, San Diego, CA, USA). The data was used to fit either a four-parameter or bi-logistic equation for each of the compound profiles, with the bottom parameter constrained to zero. Best fit curves were the subsequently used for further rate-based analysis. Due to A. succinogenes having an inherent nature to produce biofilm, spectrophotometric determination of biomass accumulation only proved useful until such time that flocculation occurred. Thus, DCW measurements of the final biomass concentration was measured in triplicate and corrected for volume chances due to $\mathrm{NaOH}$ and Antifoam addition. This measurement served as an upper limit constraint when fitting the logistic growth curves. Rate-based mass and redox balances were performed to asses closure and accuracy of metabolite measurement. Mass balances were performed by calculating the stoichiometric amount of dextrose needed to satisfy the production of biomass and catabolites measured, and comparing this to the change in the measured amount of dextrose. Similarly, redox balances were performed by calculating the stoichiometric amounts redox produced and consumed from the given catabolites.

\section{Conclusions}

A. succinogenes displayed high selectivity toward utilising its most ATP efficient catabolic routes in preference to less efficient routes over the entire course of its fermentative cultivation, with the exception of pyruvate excretion, irrespective of media composition. The peak in pyruvate excretion was found to coincide with the termination of growth, as well as being proximate to the peak of the PDH route. Due to the low energy-extraction efficiency of the pyruvate excretion route, it is postulated that the activation of this route is not energy driven as with the other routes, but rather a mechanism employed by the organism to divert carbon away from the production of acetic acid. This is emphasised by the lack of measured oxaloacetic acid production, which would, in theory, result in a similar energy efficiency to pyruvate excretion.

It was also found that $A$. succinogenes uses its liquid environment to its advantage in order to obtain an initial brief period of high energy extraction efficiency with the onset of growth. This was done by channeling reduction power generated through acetic acid production to the media until the media's oxidative capabilities were exhausted. This was found to be at a media ORP of approximately $-370 \mathrm{mV}$ and coincided with the onset of flocculation of biomass and initialisation of the PDH energy extraction route.

Pure homosuccinic acid production via the OPPP pathway was found to be active during the final stages of fermentation. This was attributed to the relatively low energy extraction efficiency of the pathway. Therefore, it may be possible to isolate this behaviour; however, for A. succinogenes to reach this stage it first must exhaust all other, more energyefficient routes.

Author Contributions: Conceptualization, W.G.L. and W.N.; methodology, W.G.L. and W.N.; software, W.G.L. and S.C.M.; validation, W.G.L., S.C.M., H.G.B. and W.N.; formal analysis, W.G.L., S.C.M., H.G.B. and W.N.; investigation, W.G.L.; resources, H.G.B. and W.N.; data curation, W.G.L.; writing—original draft preparation, W.G.L., S.C.M., H.G.B. and W.N.; writing—review and editing, W.G.L., S.C.M., H.G.B. and W.N.; visualization, W.G.L.; supervision, H.G.B. and W.N.; project administration, W.G.L. and W.N.; funding acquisition, H.G.B. and W.N. All authors have read and agreed to the published version of the manuscript. 
Funding: This research received no external funding.

Data Availability Statement: The data presented in this study are openly available in the University of Pretoria Research Data Repository at DOI: 10.25403/UPresearchdata.16398081.

Conflicts of Interest: The authors declare no conflict of interest.

\section{References}

1. Guettler, M.; Rumler, D.; Jain, M. Actinobacillus succinogenes sp. nov., a novel succinic-acid-producing strain from the bovine rumen. Int. J. Syst. Bacteriol. 1999, 49, 207-216. [CrossRef] [PubMed]

2. Lin, S.K.C.; Du, C.; Koutinas, A.; Wang, R.; Webb, C. Substrate and product inhibition kinetics in succinic acid production by Actinobacillus succinogenes. Biochem. Eng. J. 2008, 41, 128-135. [CrossRef]

3. McKinlay, J.B.; Laivenieks, M.; Schindler, B.D.; McKinlay, A.A.; Siddaramappa, S.; Challacombe, J.F.; Lowry, S.R.; Clum, A.; Lapidus, A.L.; Burkhart, K.B.; et al. A genomic perspective on the potential of Actinobacillus succinogenes for industrial succinate production. BMC Genom. 2010, 11, 680. [CrossRef] [PubMed]

4. Global Maleic Anhydride Market Size Analysis Report, 2019-2025. Available online: https:/ /www.grandviewresearch.com/ industry-analysis/maleic-anhydride-market (accessed on 15 July 2021).

5. Werpy, T.; Petersen, G.R. Top Value Added Chemicals from Biomass. Volume 1-Results of Screening for Potential Candidates from Sugars and Synthesis Gas; National Renewable Energy Laboratory: Golden, CO, USA, 2004.

6. Bozell, J.J.; Petersen, G.R. Technology development for the production of biobased products from biorefinery carbohydrates-the US Department of Energy's "Top 10" revisited. Green Chem. 2010, 12, 539-554. [CrossRef]

7. Jiang, Z.; Cui, Z.; Zhu, Z.; Liu, Y.; Tang, Y.; Hou, J.; Qi, Q. Engineering of Yarrowia lipolytica transporters for high-efficient production of biobased succinic acid from glucose. Biotechnol. Biofuels. 2021, 14, 145. [CrossRef] [PubMed]

8. Li, C.; Yang, X.; Gao, S.; Wang, H.; Lin, C.S.K. High efficiency succinic acid production from glycerol via in situ fibrous bed bioreactor with engineered Yarrowia lipolytica. Bioresour. Technol. 2017, 225, 9-16. [CrossRef] [PubMed]

9. Yan, Q.; Zheng, P.; Dong, J.; Sun, Z. A finrpus bed bioreactor to improve the productivity of succinic acid by Actinobacillus succinogenes. J. Chem. Technol. Biotechnol. 2013, 89, 1760-1766. [CrossRef]

10. Indera Luthfi, A.A.; Jahim, J.M.; Harun, S.; Tan, J.P.; Mohammad, A.W. Biorefinery approach towards greener succinic acid production from oil palm frond bagasse. Process Biochem. 2016, 51, 1527-1537. [CrossRef]

11. Brink, H.G.; Nicol, W. Succinic acid production with Actinobacillus succinogenes: Rate and yield analysis of chemostat and biofilm cultures. Microb. Cell Fact. 2014, 13, 111. [CrossRef] [PubMed]

12. Bradfield, M.F.A.; Nicol, W. The pentose phosphate pathway leads to enhanced succinic acid flux in biofilms of wild-type Actinobacillus succinogenes. Appl. Microbiol. Biotechnol. 2016, 100, 9641-9652. [CrossRef] [PubMed]

13. Bradfield, M.F.A.; Nicol, W. Continuous succinic acid production by Actinobacillus succinogenes in a biofilm reactor: Steady-state metabolic flux variation. Biochem. Eng. J. 2014, 85, 1-7. [CrossRef]

14. McKinlay, J.B.; Shachar-Hill, Y.; Zeikus, J.G.; Vieille, C. Determining Actinobacillus succinogenes metabolic pathways and fluxes by NMR and GC-MS analyses of ${ }^{13}$ C-labeled metabolic product isotopomers. Metab. Eng. 2007, 9, 177-192. [CrossRef] [PubMed]

15. Park, D.H.; Zeikus, J.G. Utilization of electrically reduced neutral red by Actinobacillus succinogenes: Physiological function of neutral red in membrane-driven fumarate reduction and energy conservation. J. Bacteriol. 1999, 181, 2403-2410. [CrossRef] [PubMed]

16. Herselman, J.; Bradfield, M.F.A.; Vijayan, U.; Nicol, W. The effect of carbon dioxide availability on succinic acid production with biofilms of Actinobacillus succinogenes. Biochem. Eng. J. 2017, 117, 218-228. [CrossRef]

17. Villadsen, J.; Nielsen, J.; Lidén, G. Bioreaction Engineering Principles, 3rd ed.; Springer: New York, NY, USA, 2011; ISBN 978-1-44199687-9.

18. Li, J.; Jiang, M.; Chen, K.-Q.; Ye, Q.; Shang, L.-A.; Wei, P.; Ying, H.-J.; Chang, H.-N. Effect of redox potential regulation on succinic acid production by Actinobacillus succinogenes. Bioprocess Biosyst. Eng. 2010, 33, 911-920. [CrossRef] [PubMed]

19. Walter, A.; Gutknecht, J. Permeability of small nonelectrolytes through lipid bilayer membranes. J. Membr. Biol. 1986, 90, 207-217. [CrossRef] [PubMed]

20. McKinlay, J.B.; Zeikus, J.G.; Vieille, C. Insights into Actinobacillus succinogenes fermentative metabolism in a chemically defined growth medium. Appl. Environ. Microbiol. 2005, 71, 6651-6656. [CrossRef] [PubMed] 